\title{
Development of a Steady-State Thermodynamic Model in Microsoft Excel for Performance Analysis of Industrial Gas Turbines
}

\author{
Tyler A. Clifford, Samuel Cruz-Manzo*, Yu Zhang \\ School of Engineering \\ University of Lincoln \\ Lincoln, United Kingdom \\ *scruzmanzo@lincoln.ac.uk
}

\author{
Vili Panov, Anthony Latimer \\ Siemens Industrial Turbomachinery \\ Lincoln, United Kingdom
}

\begin{abstract}
In this paper, an off-design performance prediction model for a single shaft industrial gas turbine (IGT) using Microsoft Excel with Visual Basic for Applications (VBA) programming is presented. The modelling architecture is comprised of fundamental thermodynamic equations describing the performance of IGTs. A graphical user interface has been constructed to allow an easy interaction of the model to predict IGT performance at different operating conditions. Component characteristic maps for the compressor and turbine with a bilinear interpolation method have been implemented in the Excel modelling architecture. A commercial thermodynamic toolbox (Thermolib, EUtech Scientific Engineering GmbH) which is compatible with Simulink environment has been considered to validate Excel model of the IGT system. This Excel modelling architecture could be a valuable reference tool for engineers and students to understand IGT performance at different ambient and operating conditions.
\end{abstract}

Keywords-Gas Turbine; Excel Model; Thermodynamics

\section{INTRODUCTION}

Industrial gas turbines (IGTs) have been used for power generation since 1939 [1] and are now one of the most common methods for generating electricity all over the world by converting mechanical energy to electrical energy from a rotating shaft. Other applications of gas turbines include jet engines for the aerospace industry as well as mechanical drivers in the oil and gas industry where industrial gas turbines can be used to drive either a pump or compressor.

Many computer programs have been developed to predict the performance of gas turbines outside of its design operating point, referred to as off-design performance. Key off-design performance methodologies have been presented by Walsh and Fletcher [2], Saravanamuttoo [3] and Razak [4] for the performance prediction of generic gas turbines. There have been efforts to improve these methods and implement them to predict the performance of a specific manufacturer's engine, for example, Rashidzadeh et al. [5] developed a model to predict the steady state performance of a Siemens SGT-600 industrial twin shaft gas turbine for mechanical drive applications that was validated against real life data. Gobran [6], has also developed an off-design performance prediction of a Solar Centaur-40 gas turbine using Simulink without the manufacturers' component maps, where a scaling method was incorporated to adapt generic component maps to match those of the Centaur-40 specification.

The off-design performance of an engine is considered when the engine is running outside of its design conditions for operating point defined with turbine speed and power output. During an engine lifetime, it will almost certainly run at conditions outside of its design operating point. There are many possibilities that can affect the running point of a gas turbine such as the geographical location that may have ambient conditions of a much greater or lower ambient temperature or may be operating at a significant altitude above sea level, again affecting the ambient temperature and pressure. In order to determine the overall steady state performance, the operating point must be determined on each component, to ensure a mass flow and energy balance.

In this paper, a performance prediction model of a single shaft gas turbine has been developed in Microsoft Excel. It acts as a reference tool for the performance analysis of IGTs and acts as a foundation for larger, more complex models to be developed in the future, demonstrating what can be achieved without the need for more advanced, expensive software. Fig. 1 shows the main components comprising a single shaft IGT. The basic gas turbine thermodynamic cycle consists of four stages.

\section{Modelling ARCHITECTURE}

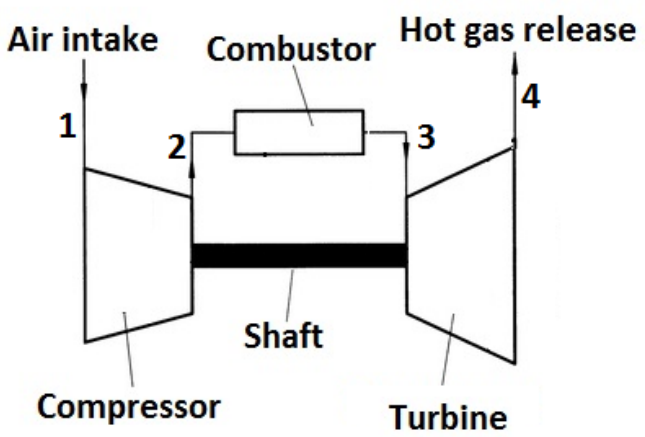

Fig. 1. Single shaft IGT 
The first stage begins at the inlet of the compressor which draws in ambient air and increases the pressure across the compressor, which also increases the temperature of the air. The high-pressure air then enters the combustion chamber where it is mixed with fuel, typically natural gas, and burned. The high temperature air and fuel mixture leave the combustion chamber and enter the turbine inlet where they are then expanded across the back to atmospheric pressure. The compressor is driven by the turbine through a mechanical system (shaft-bearing). The rotational speed of the compressor and turbine are equal as they are directly coupled.

The following assumptions have been considered in the modelling architecture:

- Only ambient and dry conditions are considered in the thermodynamic modelling architecture. A future work will consider logarithm polynomial functions of thermodynamic properties as a function of different ambient conditions.

- $\quad$ No pressure losses in the inlet ducting and exhaust are considered, hence the exit pressure from the turbine is equal to the atmospheric pressure at the inlet of compressor $(P 4=P 1)$.

- The pressure of gas leaving the combustor chamber has been defined to be $2 \%$ less than pressure discharged by compressor $P_{3}=P_{2} * 0.98$.

- The turbine inlet temperature and pressure is equal to the temperature and pressure of gas leaving the combustor.

- The fuel is only methane $\mathrm{CH}_{4}$ with a lower heating value (LHV) of $48120 \mathrm{~kJ} / \mathrm{kg}$ [2] and is assumed that it has entirely reacted and consumed during the combustion process.

The compressor requires a map that relates rotational speed, pressure ratio, mass flow rate, and efficiency, as shown in Fig. 2. A turbine map that relates rotational speed, pressure ratio, mass flow rate, and efficiency is required as well. The maps calculate the mass flow rate and efficiency from the rotational speed and the pressure ratio which act as inputs. The performance maps for compressor and turbine presented in this study have been scaled to develop the Excel modelling tool. Once completed the model, it will be possible to implement specific compressor and turbine performance maps.

\section{A. Thermodynamic calculations for off-design performance}

The off-design performance prediction for a single shaft gas turbine is based on the equations proposed by Saravanamuttoo [7] for the mass flow balance between the compressor and turbine. The addition of fuel into the combustor module is considered, with a bi-linear interpolation method for determining the values for flow, pressure ratio and efficiency at any defined speed. Since the tedious nature of off-design prediction models it is almost impossible to implement efficiently without the use of a computer program, hence most models are constructed within specialized software environment using available catalogue data for component characteristics and thermodynamic properties of the working fluid. In this study, the modelling architecture is constructed within Microsoft Excel VBA, therefore making the prediction model readily available to engineers in industry, or for ease of use in an educational environment. The values for compressor

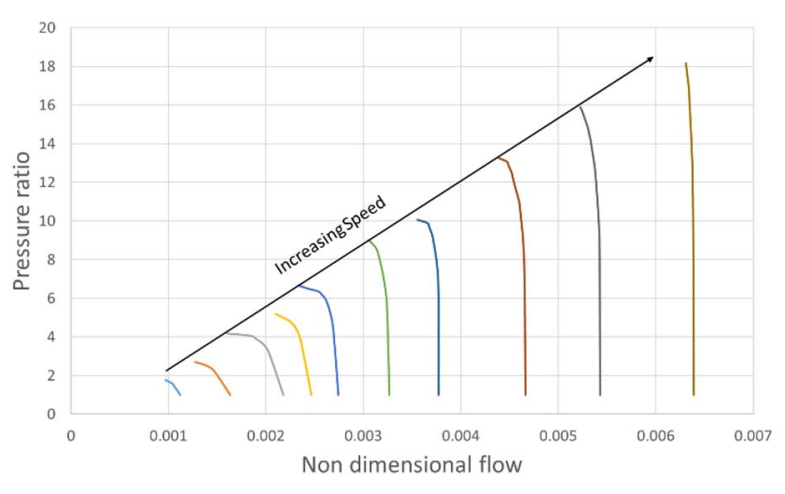

Fig. 2. Compressor performance map

non-dimensional flow and pressure ratio are obtained from the compressor map using bilinear interpolation. Interpolation method in performance maps of compressor and turbine will be discussed in the next section. The thermodynamic equations for the performance of the compressor are defined from Eqs. 1-5:

$$
\text { Compressor_nondimensional_speed }=\frac{N}{\sqrt{T_{1}}}
$$

where $N$ is the speed required (rpm) and $T_{1}$ is the ambient temperature $(\mathrm{K})$. The compressor exit parameters $P_{2}, T_{2}$, flow $W_{l}$, and the power absorbed by the compressor $c_{\text {pow }}$ are calculated as follows:

$$
\begin{gathered}
P_{2}=P_{1} * P_{R} \\
T_{2}=T_{1}+\frac{T_{1}}{\eta_{C}}\left(P_{R C} \frac{\gamma-1}{\gamma}-1\right) \\
W_{1}=\frac{\text { Compressor } \_ \text {non dim ensional_flow } * P_{1}}{\sqrt{T_{1}}} \\
c_{\text {pow }}=W_{1} C_{P}\left(T_{2}-T_{1}\right)
\end{gathered}
$$

where $P_{R C}$ is the compressor pressure ratio and $\eta_{C}$ is the efficiency respectively obtained from the compressor map interpolation, $W_{1}$ is the air flow, and $C_{P}$ is the specific heat capacity. The compressor non-dimensional flow is the value obtained from the compressor map interpolation. The interpolation method will be discussed in the next section. The value of the turbine inlet temperature is estimated, the fuel to air ratio $F A R$, fuel flow $m f$, combustor pressure and turbine pressure ratio can then be calculated from the following equations:

$$
\begin{gathered}
F A R=\frac{C_{P}\left(T_{3}-T_{2}\right)}{L H V} \\
m f=W_{1}^{*} F A R \\
P_{3}=P_{2}-\left(P_{2}^{*} * 0.02\right) \\
P_{R t}=\frac{P_{3}}{P_{4}}
\end{gathered}
$$

where LHV is the lower heating value of the fuel Methane, $T_{3}$ is the estimated turbine inlet temperature and $P_{R t}$ is the pressure ratio across the turbine where $P_{4}$ is equal to the 
ambient pressure $P_{l}$. Noting that it is assumed a pressure drop of $2 \%$ across the combustor. From the turbine pressure ratio calculated in Eq. 9 and the turbine non-dimensional speed, the flow and efficiency can be determined from the turbine characteristic map.

$$
\text { Turbine _non_dim ensional _ speed }=\frac{N}{\sqrt{T_{3}}}
$$

where $N$ is the speed required, as the engine is single shaft configuration the rotational speed of the compressor and turbine are equal as they are directly coupled.

Turbine_non_dimensional_flow $=\frac{W_{1} \sqrt{T_{1}}}{P_{1}} * \frac{P_{1}}{P_{3}} * \sqrt{\frac{T_{3}}{T_{1}}} * \frac{W_{3}}{W_{1}}$

and $W_{3}$ is, the flow leaving the combustion chamber and entering the turbine, which is the sum of the air flow from the compressor and the fuel flow into the combustor. The turbine non-dimensional flow is calculated and compared with the non-dimensional flow obtained from the turbine map characteristic. Once the turbine non-dimensional flow error is $0.5 \%$ or less the turbine exit temperature, $T_{4}$, turbine power tpow, net power output pnet, specific fuel consumption $S F C$ and thermal efficiency, $\eta_{t h}$ can be calculated from the following equations:

$$
\begin{gathered}
T_{4}=T_{3}-\left(T_{3} * \eta_{t}\right)\left(1-{\frac{1}{P_{R t}}}^{\frac{\gamma-1}{\gamma}}\right) \\
\text { tpow }=W_{3} * C_{P}\left(T_{3}-T_{4}\right) \\
\text { pnet }=\text { tpow }- \text { cpow } \\
\text { SFC }=3600 *\left(\frac{m f}{p n e t}\right) \\
\eta_{t h}=\frac{\text { pnet }}{m f * L H V} * 100
\end{gathered}
$$

where, $\eta_{t}$ is the turbine efficiency from the turbine characteristic map. The thermal efficiency, $\eta_{t h}$ is the percentage of power output for fuel energy input.

\section{B. Bi-linear Interpolation of component maps in Excel}

The interpolation of component characteristic maps is vital in the off-design performance prediction of IGTs. The thermodynamic equations defining the off-design performance of IGTs have been discussed in previous section.

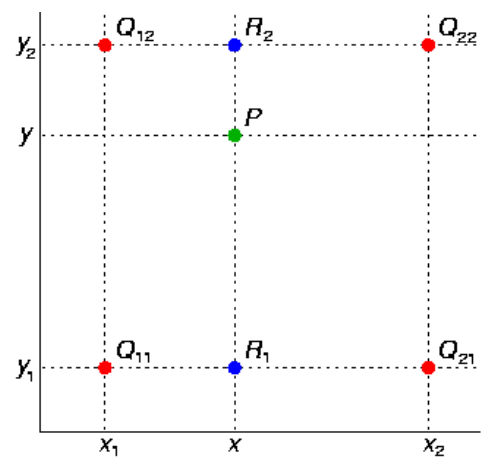

Fig. 3. Bi-linear interpolation
A bilinear interpolation method [8] for the compressor characteristic map has been considered in the modelling architecture. The turbine flow and efficiency map used in this study is based on fixed turbine efficiency and flow characteristics, which is a function of pressure ratio only, therefore, only a simpler linear interpolation was applied. Bilinear interpolation is more commonly used as a resampling method in image processing. When an image is resized the pixels get 'stretched', therefore, a new pixel is added in between four existing pixels, where the colour or intensity is determined based on the weighted average of the surrounding four pixels, or as known as bilinear interpolation. The component maps for the compressor and turbine are tabulated in an excel spreadsheet file with non-dimensional flow, pressure ratio and efficiency in descending order for each corresponding constant speed line. The interpolation is carried out between two adjacent columns for non-dimensional flow, pressure ratio, and efficiency to find the weighted average between four points, in this case, non-dimensional flow, pressure ratio or efficiency. Fig. 3 shows the points used on a 2D grid for bilinear interpolation, where the $y$ values are line number used on the compressor map shown in Fig. 4. The $x$ values are the values for speed and the $Q$ values are the values for either non-dimensional flow, pressure ratio or efficiency in compressor map. The turbine characteristic map can also be represented with graphic representation lines for the bilinear interpolation method. The values of $R_{1}, R_{2}$ and $P$ for the bilinear interpolation represented in Fig. 3 can be determined from the following equations [8]:

$$
\begin{aligned}
& R_{1}=\left(\frac{x_{2}-x}{x_{2}-x_{1}}\right) Q_{11}+\left(\frac{x-x_{1}}{x_{2}-x_{1}}\right) Q_{21} \\
& R_{2}=\left(\frac{x_{2}-x}{x_{2}-x_{1}}\right) Q_{12}+\left(\frac{x-x_{1}}{x_{2}-x_{1}}\right) Q_{22} \\
& P_{1}=\left(\frac{y_{2}-y}{y_{2}-y_{1}}\right) R_{1}+\left(\frac{y-y_{1}}{y_{2}-y_{1}}\right) R_{2}
\end{aligned}
$$

The modelling architecture uses functions to look up the required values to perform the interpolation, largely a combination of one index function and either one or two match functions.

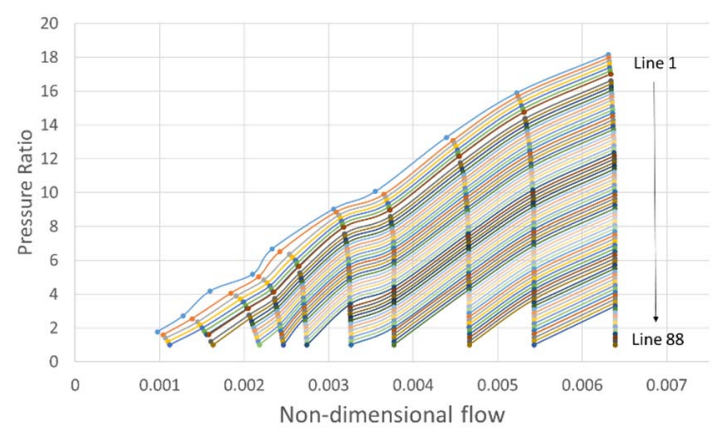

Fig. 4. Graphical representation of lines used for compressor map interpolation 


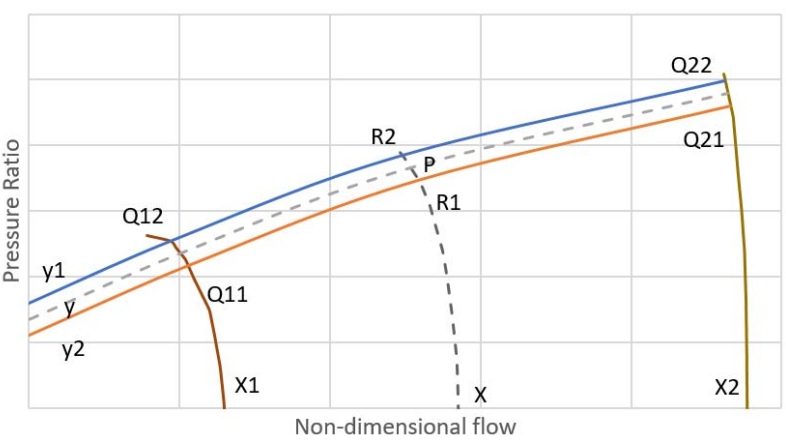

Fig. 5. Bilinear Interpolation in Compressor Flow Map

The match functions simply look up a range of values and determines the location of the nearest value and the index functions returns the actual numerical value at this location. An example of how the combination of the index and match functions is:

\section{Value $=$ INDEX $($ Range of values, MATCH(lookup_y, Lookup_range, type of match), MATCH(lookup_x, lookup_range, type of match)}

where the index range of values is the range in which the value is to be obtained from, so in this case either nondimensional flow, pressure ratio or efficiency. The lookup_y value is the value of line used for interpolation and the lookup_x value the value of non-dimensional speed, with the lookup_range is the two ranges containing the value of line for interpolation or non-dimensional speed. The type of match can either be 1,0 or -1 where 1 returns the largest value less than or equal to the match lookup value, 0 returns an exact match and -1 returns the smallest value that is equal to or greater than the match lookup value.

\section{Bilinear interpolation in Compressor characteristic map}

Firstly, the compressor non-dimensional speed is calculated from the required rotational speed divided by the square root of the ambient temperature as shown in Eq. 1 and the values for speed in the tabulated tables of non-dimensional flow and pressure ratio are made non-dimensional. The lines produced from the tabulated data are labelled in increments of one, however data is interpolated in increments of 0.1 , therefore, ten values of non-dimensional flow and pressure ratio between each line to improve the accuracy of matching the power to the required power. It is required to specify the required power in the gas turbine model, therefore the model will determine what the operating point is to produce the required power using an iterative technique, sequentially increasing the value of the line of values used by 0.1 until the powers agree. The value of the line of data required is looked up and the two closest matches selected, so for a line value of 10.2, the data from line 10 and 11 will be used for as the values of $y$ in the bilinear interpolation, as well as the two closest matches for non-dimensional speed as the values of $\mathrm{x}$,

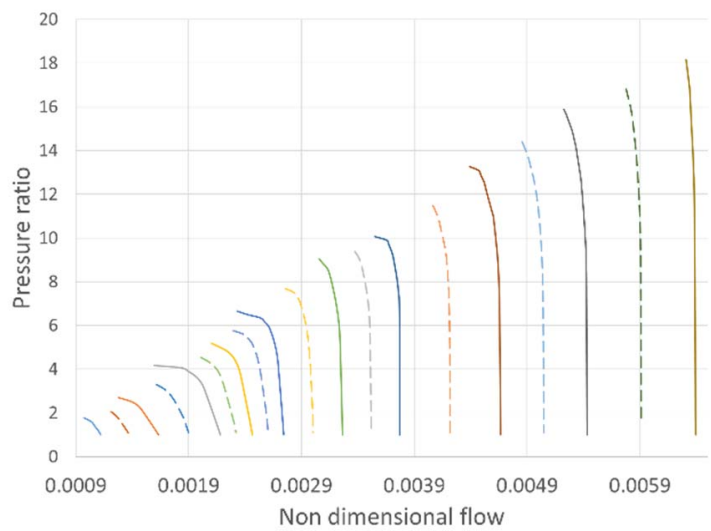

Fig. 6. Predicted (dashed) constant speed lines from bilinear interpolation method

one larger and one smaller than the required speed line, as shown in Fig. 5. The values for non-dimensional flow and pressure ratio can now be gathered for points Q12, Q22, Q11 and Q21. Eqs. 17, 18 and 19 are then implemented to determine the weighted average between the four points of either non-dimensional flow or pressure, the system thermodynamic calculations are then performed to determine the net power at the current operating point, and the process is repeated until the difference between the net power and the required power is less than or equal to $0.01 \%$.

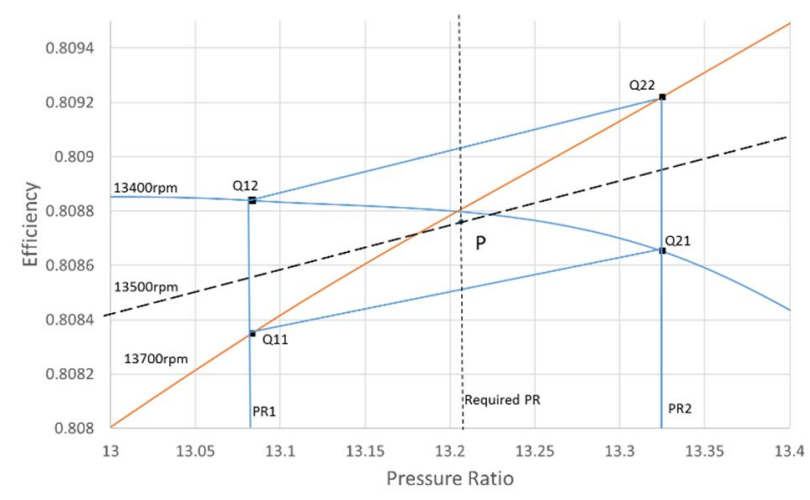

Fig. 7. Bilinear Interpolation in Compressor Efficiency Map

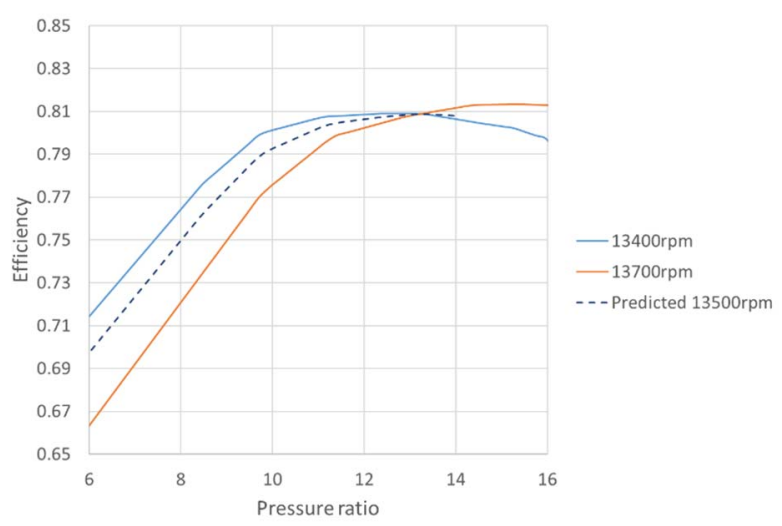

Fig. 8. Predicted efficiency at $13500 \mathrm{rpm}$ using bilinear interpolation method 


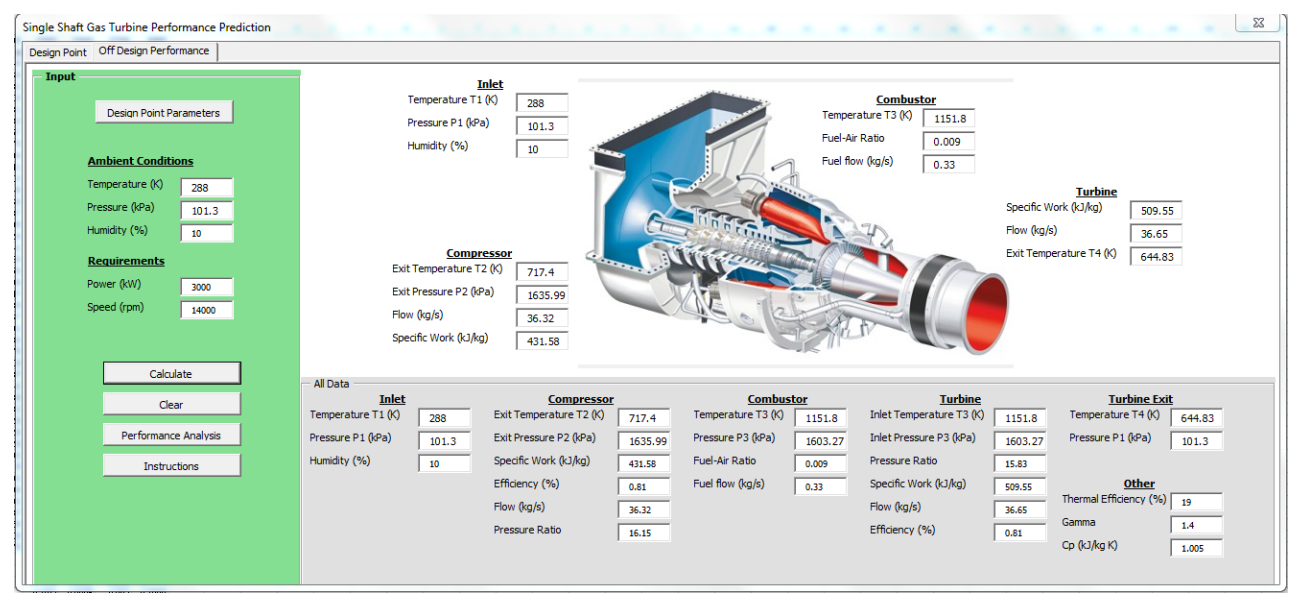

Fig. 9. Graphic Interface for Model execution

Once the pressure ratio has been determined from the compressor characteristic then the efficiency can be determined from interpolation of compressor efficiency map by looking up the two closest values of pressure ratio, as shown in Fig. 7, one greater and one lower and the same for non-dimensional speed, therefore four values of compressor efficiency are selected, a bilinear interpolation is then performed, first to determine the compressor efficiency as the correct pressure ratio and secondly to determine the compressor efficiency at the required speed. The interpolation of the compressor component map allows for the prediction of flow and pressure ratio for various power requirements and at different ambient conditions outside of the available data. Figs. 6 and 8 show the predicted constant speed lines for flow and efficiency maps using the bilinear interpolation method. This method can be applied to turbine component maps if the flow and efficiency characteristic are a function of both speed and pressure ratio, this will be considered in the further work.

\section{Graphic Interface for IGT simulation}

A graphic interface has been constructed to define inputs and display outputs of the IGT at different power required. The implemented model requires to define ambient conditions such as temperature and pressure as well as the power and rotational speed required during IGT operation. The flow discharged by compressor as well as compressor efficiency are calculated from compressor map and bilinear interpolation method as previously discussed. The temperature discharged by the compressor is calculated from estimated efficiency and thermodynamic equations. The temperature of combustion products is calculated through a balance of thermal energies and considering LHV of gas Methane. The turbine flow and efficiency are determined from the turbine maps using the turbine pressure ratio and linear interpolation. The estimated net power is compared with the required power which acts as input. When the error between estimated values from model and required output (power) is less than $0.01 \%$, the model iteration finishes and the results are displayed through the graphic interface shown in Fig. 9. The thermodynamic properties such as temperature, specific work at each stage of the IGT system are calculated. The overall thermal efficiency and fuel consumption during IGT performance are estimated as well.

The execution of the model is shown in Fig. 10 and is summarized as follows:

1. Define required speed, power, fuel and ambient conditions, $P_{1}, T_{1}$.

2. Get values of flow, pressure ratio and efficiency from compressor map.

3. Calculate $P_{2}, T_{2}$, air flow and compressor power absorbed.

4. Calculate FAR, fuel flow, $P 3$ and turbine pressure ratio (note $P_{4}=P_{1}$ ).

5. From turbine pressure ratio and speed, determine turbine flow and efficiency from turbine map.

6. Calculate turbine non-dimensional flow and compare to value obtained from map.

7. Calculate $T_{4}$, turbine power, net power output and thermal efficiency.

8. Compare required power to net power output.

9. Finish and display results.

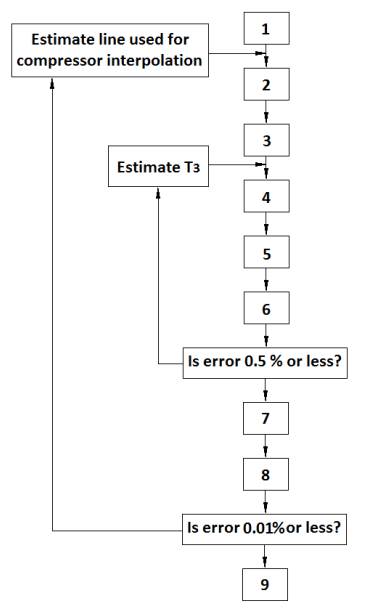

Fig. 10. Flow chart describing overall Excel Model Execution 


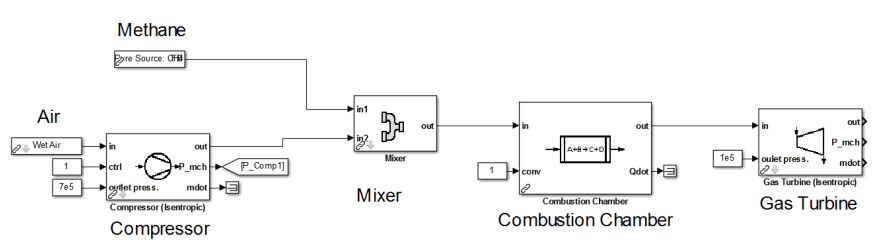

Fig. 11. Modelling architecture in Thermolib-Simulink for Excel model validation

\section{MODEL VALIDATION}

The predicted temperature across the IGT system from the Excel model is compared with the temperature predicted by a validated Simulink Model. The Simulink Model considered a thermodynamic commercial toolbox (Thermolib, EUtech Scientific Engineering GmbH) [9]. The Compressor, Combustor, and Turbine are components defined in Thermolib-Simulink library.

One of the advantages of the Thermolib library is the fact that each component (e.g. compressor, reactor, turbine, etc.) can calculate thermodynamic properties such as enthalpy, heat capacity, molar composition in a two phase-flow composition. In a previous study [10], a thermodynamic model of an IGT constructed from Thermolib library has been validated with experimental gas turbine data. The thermodynamic model constructed from Thermolib as shown in Fig. 11 assisted in the thermodynamic validation of the Excel model. The ambient conditions for the validation exercise considered ambient conditions with $T a m b=15$ $\operatorname{deg} C, P a m b=101.3 \mathrm{kPa}$, and $0 \% \mathrm{RH}$. Three operating points were considered to compare the parameters between Excel and Thermolib models. The compressor pressure ratio was increased at a constant speed of $14000 \mathrm{rpm}$. The increase in pressure ratio with constant speed yields to a reduction in air flow discharged by the compressor.

Fig. 12 shows that when the pressure ratio in the compressor is increased, the temperature of air discharged by the compressor increases. The increase in pressure ratio also yields to an increase of compressor power consumption, as shown in Fig. 12. Fig. 12 also demonstrates that the Excel model can predict the increase in air temperature discharged by the compressor. The maximum absolute error between the Excel model and Thermolib model for compressor discharge temperature is $2.047 \%$.
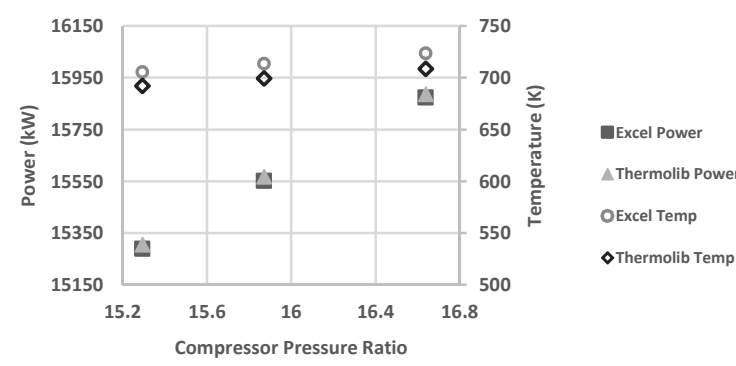

OExcel Temp

$\diamond$ Thermolib Temp

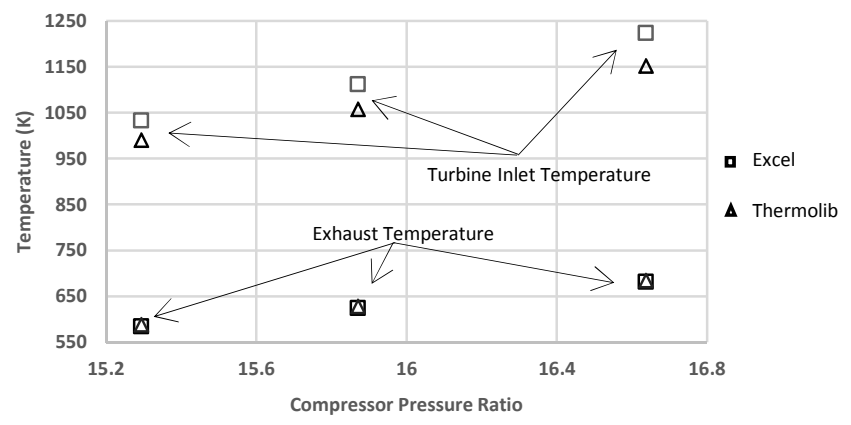

Fig. 13. Comparison between inlet temperature and exahust temperarure from turbine predicted by Excel and Thermolib

The error for compressor discharged temperature can be attributed to the fact that a constant heat capacity of fluid was considered in the Excel model. The heat capacity should be defined as a polynomial depending on temperature. This will be addressed in a future work.

Fig. 13 shows a comparison between the turbine inlet temperature predicted by Excel and Thermolib. The turbine inlet temperature is assumed to be the same as the temperature during combustion of fuel/air. The turbine inlet temperature increases with increasing pressure ratio. This is attributed to an increase in fuel demand with increasing compressor pressure ratio. A difference between enthalpy change during combustion defined in each model could yield the difference in turbine inlet temperature as shown in Fig. 13. The temperature of exhaust gas released by the turbine and predicted by Excel and Thermolib models was compared as well, as shown in Fig 13. The maximum absolute error between the Excel model and Thermolib model for turbine exit temperature is $0.576 \%$.

Fig. 14 shows the net power and thermal efficiency of the IGT system predicted by Excel and Thermolib. The net power is the difference between turbine power and compressor power. The thermal efficiency and net power increase with increasing the compressor pressure ratio. The maximum absolute error between the Excel model and Thermolib model for thermal efficiency is $1.56 \%$. A difference between calorific value of fuel defined in each model could yield the difference in thermal efficiency.

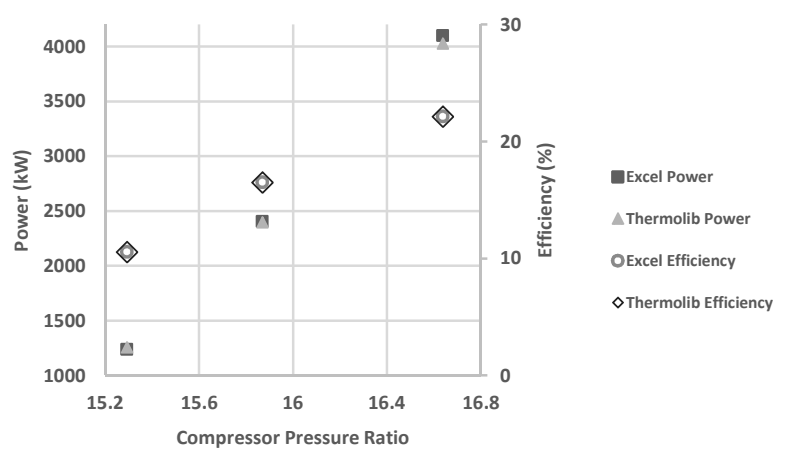

Fig. 14. Comparison between Net power and Thermal efficiency predicted by Excel and Thermolib
Fig. 12. Comparison between temperature and power from compressor predicted by Excel and Thermolib 


\section{CONCLUSIONS}

A thermodynamic model of a single shaft IGT has been developed in Microsoft Excel with VBA programming. It could be used as a reference tool for the performance analysis of IGTs at different operating conditions. Bilinear interpolation in component maps allowed the estimation of fluid flow rate and efficiencies at different IGT operating conditions (pressure ratio, rotational speed). The simulated temperature of the IGT system predicted by the Excel model was compared with the simulated temperature predicted by a previously validated Simulink thermodynamic model at three different operating points. The model developed in this study is a first attempt at modelling development in a single shaft IGT considering Microsoft Excel. In future work emissions such as NOx, CO or UHC (unburned hydrocarbons) resulting from the fuel-air combustion will be considered. Further modelling work will also address the effect of ambient conditions on the IGT performance.

\section{ACKNOWLEDGMENT}

This work was supported by Siemens Industrial Turbomachinery, Lincoln, U.K.

\section{REFERENCES}

[1] Wartsila, "Gas turbine for power generation: Introduction," 2017. [Online]. Available: http://www.wartsila.com/energy/learningcenter/technical-comparisons/gas-turbine-for-power-generationintroduction. [Accessed March 2017].)

[2] P. F. Philip P. Walsh, Gas Turbine Performance, Blackwell Science Ltd, 2004.

[3] H. I. H. Saravanamuttoo, Gas turbine theory, Prentice Hall, 2008.

[4] A. M. Y. Razak, "Industrial Gas Turbines: Performance and operability," Woodhead Publishing Limited, Cambridge, 2007.

[5] H. Rashidzadeh, S. M. Hosseinalipour, and A. Mohammadzadeh, "The SGT-600 industrial twin-shaft gas turbine modeling for mechanical drive," Journal of Mechanical Science and Technology, vol. 29, no. 10, pp. 4473-4481, 2015.

[6] M. Gobran, "Off-design performance of Solar Centaur-40 gas turbine engine using simulink," Ain Shams Engineering Journal, vol. 4, pp. 285-298, 2013.

[7] G. R. H. C. P. S. HIH Saravanamuttoo, Gas Turbine Theory, Harlow: Pearson Education Ltd., 2009.

[8] The supercomputing blog, "Coding bilinear interpolation," 30 December 2011. [Online]. http://supercomputingblog.com/graphics/coding-bilinearinterpolation/. [Accessed 20 January 2017].

[9] Thermolib 5.3, EUtech Scientific Engineering GmbH. Aachen, Germany.

[10] S. Cruz-Manzo, et al. "A thermodynamic transient model for performance analysis of a twin shaft industrial gas turbine", Proceedings of ASME Turbo Expo 2017, GT2017-64376, June 2630, Charlotte, NC, USA. 